Low Light Adaptive Correction in Support of the Space Situational (DCT-FY08)

Mark Chun

UNIVERSITY OF HAWAII SYSTEMS HONOLULU

$12 / 09 / 2014$

Final Report

DISTRIBUTION A: Distribution approved for public release.

Air Force Research Laboratory

AF Office Of Scientific Research (AFOSR)/ RSE

Arlington, Virginia 22203

Air Force Materiel Command 


\section{REPORT DOCUMENTATION PAGE}

Form Approved

OMB No. 0704-0188

The public reporting burden for this collection of information is estimated to average 1 hour per response, including the time for reviewing instructions, searching existing data sources, gathering and maintaining the data needed, and completing and reviewing the collection of information. Send comments regarding this burden estimate or any other aspect of this collection of information, including suggestions for reducing the burden, to Department of Defense, Executive Services, Directorate (0704-0188).

Respondents should be aware that notwithstanding any other provision of law, no person shall be subject to any penalty for failing to comply with a collection of information if it does not display a currently valid OMB control number.

PLEASE DO NOT RETURN YOUR FORM TO THE ABOVE ORGANIZATION.
1. REPORT DATE (DD-MM-YYYY)
2. REPORT TYPE
08-12-2014

4. TITLE AND SUBTITLE

LOW LIGHT ADAPTIVE CORRECTION IN SUPPORT OF THE SPACE SITUATIONAL

AWARENESS MISSION (DCT-FYO8)
3. DATES COVERED (From - TO)

01-03-2008 to 30-11-2011

5a. CONTRACT NUMBER

5b. GRANT NUMBER

FA9550-08-1-0113

5c. PROGRAM ELEMENT NUMBER

5d. PROJECT NUMBER

6. AUTHOR(S)

Mark Chun

5e. TASK NUMBER

5f. WORK UNIT NUMBER

7. PERFORMING ORGANIZATION NAME(S) AND ADDRESS(ES)

UNIVERSITY OF HAWAII SYSTEMS HONOLULU

8. PERFORMING ORGANIZATION

2530 DOLE STREET SAK D-200

REPORT NUMBER

HONOLULU, HI 96822-2309 US

9. SPONSORING/MONITORING AGENCY NAME(S) AND ADDRESS(ES)

AF Office of Scientific Research

875 N. Randolph St. Room 3112

Arlington, VA 22203

AFOSR

11. SPONSOR/MONITOR'S REPORT NUMBER(S)

\section{DISTRIBUTION/AVAILABILITY STATEMENT}

A DISTRIBUTION UNLIMITED: PB Public Release

\section{SUPPLEMENTARY NOTES}

\section{ABSTRACT}

Numerous wavefront sensing approaches are used in current adaptive optics systems. Each of these has features that make each attractive under certain situations. Roddier curvature (RC) adaptive optics systems use a sensor that measures the second derivative of the wavefront phase. Its use is common in astronomical adaptive optics systems and it has two key advantages: (1) a minimum number of sensors is required per wavefront sensor subaperture allowing the use of photon-counting devices and operations in very low-light level and (2) an "optical gain" within the wavefront sensor that allows the wavefront sensor to be tuned to existing turbulence conditions. A key question for RC adaptive optics systems is whether they remain compelling in the low-light level regime as the number of subapertures increases. This was the main avenue of study for this program.

\section{SUBJECT TERMS}

DCT, FY08

16. SECURITY CLASSIFICATION OF: a. REPORT

U

\section{b. ABSTRACT}

U c. THIS PAGE

U
17. LIMITATION OF ABSTRACT

UU
18. NUMBER OF PAGES

5 19a. NAME OF RESPONSIBLE PERSON Mark Chun

19b. TELEPHONE NUMBER (Include area code) 808-932-2317 
Final Report

\title{
Low Light Adaptive Correction in Support of the Space Situational (DCT-FY08)
}

\author{
FA9550-08-1-0113 \\ Univ. of Hawaii \\ PI: M. Chun \\ Dates of program: 3/2008-11/2010
}

\begin{abstract}
Summary
Numerous wavefront sensing approaches are used in current adaptive optics systems. Each of these has features that make each attractive under certain situations. Roddier curvature (RC) adaptive optics systems use a sensor that measures the second derivative of the wavefront phase. Its use is common in astronomical adaptive optics systems and it has two key advantages: (1) a minimum number of sensors is required per wavefront sensor subaperture allowing the use of photon-counting devices and operations in very low-light level and (2) an "optical gain" within the wavefront sensor that allows the wavefront sensor to be tuned to existing turbulence conditions. A key question for RC adaptive optics systems is whether they remain compelling in the low-light level regime as the number of subapertures increases. This was the main avenue of study for this program.

Three specific studies were made. First, we find that automating the RC wavefront sensor's optical gain to adapt to changing turbulence leads to gains over a statically set optical gain especially in highly variable turbulence conditions as can be found in tracking an object that moves quickly across the sky. Second, we derived an analytic expression for how noise in the RC sensor measurements propagates to the reconstructed phase correction. This expression compares to the previously determined case of a linear increase in the noise propagator with increasing number of subapertures in the RC wavefront sensor under the condition that the physical subaperture size remains fixed (e.g. the telescope aperture grows with number of subapertures). This suggests that RC WFSing on increasingly larger telescopes will have to contend with increasing stricter constraints on (photon) noise in the measurement. RC WFSs lose their low-light level advantage. However, the expression provides new insight into the noise propagator. In particular, in the case of a fixed telescope aperture, increasing the number of subapertures does not increase the noise propagator so for a given telescope, one is free to implement as high an order RC system as needed. Finally, we extend this work to a direct comparison between curvature and Shack-Hartmann (S-H) wavefront sensors. We find that from the noise-propagation, Shack-Hartmann gradient sensors have a distinct advantage over Roddier curvature sensors as systems are pushed to higher order correction. While the effectiveness of a given number of sensors to correct a number of modes in the wavefront aberration is different between $\mathrm{S}-\mathrm{H}$ and $\mathrm{RC}$, in the low-light level regime (where $\mathrm{RC}$ sensors historically have had an advantage), the increase in the noise propagator (and hence the wavefront reconstruction) will limit the use of high-order curvature systems.
\end{abstract}

\section{Introduction}

Adaptive optics (AO) is a technique that removes the image degradation that arises from light passing through the Earth's atmosphere. AO systems include a method to correct for the phase aberrations as well as a means to sense the distortions of the incoming light. Now deployed routinely at ground-based telescopes, AO systems enable diffraction limited imaging on ground-based telescopes as large as $10 \mathrm{~m}$ in diameter.

The motivation for this work was to explore the fundamental limitations of curvature adaptive optics 
systems in the low-light level regime for space situational awareness. Historically on astronomical telescopes curvature adaptive optics systems had advantages over other types of wavefront sensing approaches in the low-light level regime and where temporal bandwidth requirements were high. For space situational awareness, the high tracking rates and lack of bright reference sources, may benefit from curvature wavefront sensing adaptive optics.

Funding for this program enabled the group to hire Dr. Aglae Kellerer. Dr. Kellerer worked for 1.5 years exploring the fundamental limitations of curvature wavefront sensors. This work focused on the key advantages of curvature sensors - specifically the low-light limitations as higher-order systems are deployed and the use of the optical gain with the loop closed to optimize the system performance.

\section{Study 1: Automated sensitivity adjustment for a curvature sensor}

Summarized from Kellerer, Chun, and Ftaclas (2010) Applied Optics, 49(31).

Curvature wavefront sensors have a number of advantages over the more commonly used ShackHartmann (S-H) sensors. One useful characteristic that has not often been used in practice is their adjustable sensitivity to phase distortions: while $\mathrm{S}-\mathrm{H}$ sensors are designed for one fluctuation amplitude taken to be prevalent during the instrument's lifetime, the dynamic range of curvature sensors varies with the distance of the detector from the focal plane (the defocus distance). Accordingly, curvature sensors can be tuned to be optimally sensitive to the wavefront aberrations that prevail during a particular observation.

In most adaptive-optics (AO) applications, the amplitude of the phase fluctuations does indeed vary substantially. In astronomy, the seeing-a measure of the phase-fluctuation amplitudes-varies on a time scale of typically several minutes, which is shorter than the integration time of most astronomical observations. The seeing varies even faster and might become prohibitive when an observed object, such as a satellite, moves across the sky faster than astronomical targets

We have tested two different methods to automatically adjust the defocus length of a curvature sensor to the continuously changing turbulence conditions.

In the first method, the length is adjusted prior to the AO loop through the acquisition of a long- exposure image and is then kept constant. However, in extended observations of astronomical objects or in any observation of fast moving targets, such as satellites, the turbulence intensity changes over the course of the AO sequence. For such observations, we have suggested a second method to adjust the defocus length during the AO loop by use of the voltage values sent to the deformable mirror.
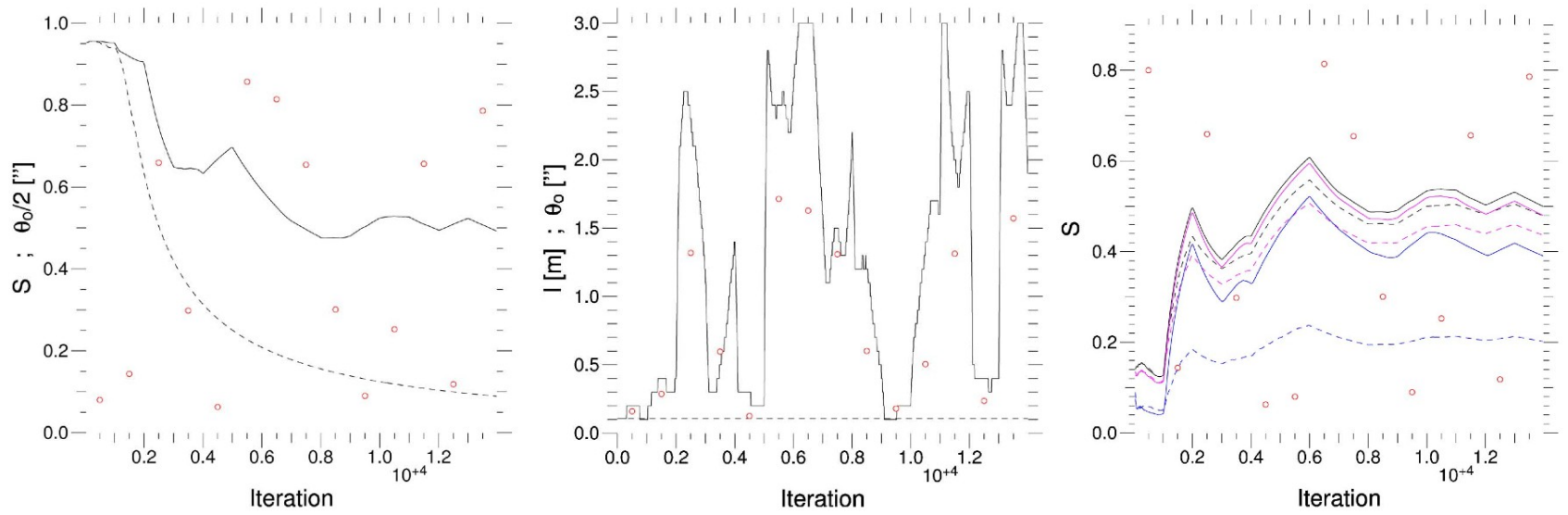

Figure 1. (Figures 5 and 6 from Kellerer, Chun, and Ftaclas 2010) - Results from numerical simulations that show the evolution of the performance metrics, Strehl (S) and full-width half-maximum $\left(\theta_{0}\right)$, as a function of iteration within the Monte-Carlo simulation. The strength of the turbulence (or seeing) was swapped between 0.1" and 1.8" every 1000 iterations for a total simulations of about 70 seconds. LEFT: The dashed curve shows the resulting Strehl when the defocus distance of the wavefront sensor was fixed at the initial 
value. The solid curve shows the resulting Strehl when the defocus distance is automatically adjusted based on the instanteous FWHM in the focal plane. The red circles are the are the FWHM. MIDDLE: The solid curve shows the optimize defocus distance (l) and circles, FWHM ( $\left.\theta_{0}\right)$. RIGHT: Solid curves, average Strehl when the defocus is adjusted during the se- quence; dashed curves, the defocus is determined prior to the AO loop when $\theta_{0}=1.6$ ". The magnitude of the reference star varies among 5 (black), 9 (magenta) and 11 (blue). Circles, $\theta_{0} / 2$. Note that as the brightness of the reference source decreases (larger magnitudes)the gain in automating the defocus distance becomes significant.

\section{Study 2: Curvature sensors: noise and its propagation}

\section{Summarized from Kellerer (2010) JOSAA, 27(11).}

The signal measured with a curvature sensor is analyzed. At the outset, we derive the required minimum number of sensing elements at the pupil edges, depending on the total number of sensing elements. The distribution of the sensor signal is further characterized in terms of its mean, variance, kurtosis, and skewness. It is established that while the approximation in terms of a Gaussian distribution is correct down to fairly low photon numbers, much higher numbers are required to obtain meaningful sensor measurements for small wavefront distortions. Finally, we indicate a closed expression for the error propagation factor and for the photon noise-induced Strehl loss.

We followed an approach developed by Hudgin's for the phase reconstruction from gradient measurements to formulate the phase reconstruction from curvature sensor measurements. To our knowledge this is the first demonstration of such an approach and one that greatly simplifies the analysis and potential optimization of curvature AO systems. Such an approach note only enables an analytic expression for the noise propagator but also sets up the framework for the incorporation of optimal phase estimators and the inclusion of the statistics of the atmospheric turbulence itself in the determination of the phase correction from the curvature sensor. We did not, however, explore these optimizations during this study.

The approach provides the noise propagator for a curvature sensor to terms of the number of sensors, the defocus distance, and the diameter of the telescope. We find that that the error propagation factor increases linearly with $\mathrm{N}$ for a unit sampling size and a constant extra-focal distance (as has been determined previously by others) and that it is almost independent of $\mathrm{N}$ when the pupil surface is constant and the extra-focal distance is adjusted (see Fig. 6 from Kellerer 2011 below).

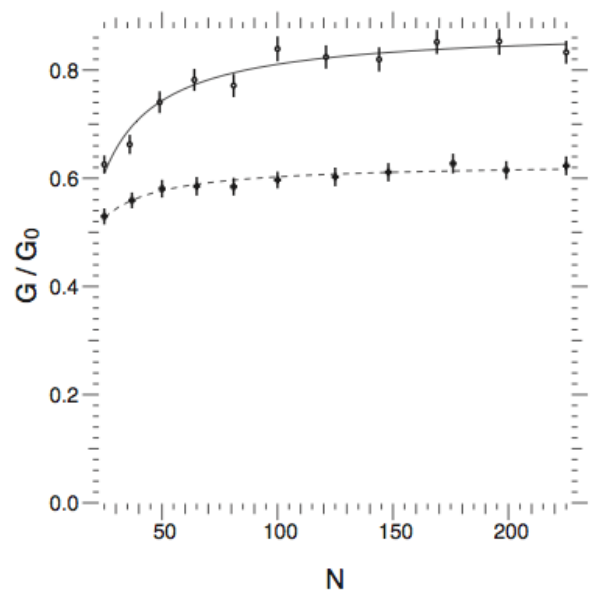

Figure 2: The error propagator's $\left(G / G_{0}\right)$ dependence on the number of sensors/subapertures. The solid curve represents a circular aperture while the dotted line represents and idealized square sensor. 
This new result is important and says that for a given telescope, there is no penalty for implementing more sensors in the curvature sensor. This in hindsight is intuitive given that the curvature sensor is operating with a noiseless detector. Namely, since there is no read-noise penalty, a curvature system is free to effectively resample the measurements and use as many degrees of freedom as it can.

\section{Study 3: Error propagation: a comparison of Shack-Hartmann and curvature sensors}

\section{Summarized from Kellerer and Kellerer (2011) JOSAA, 28(5).}

Phase estimates in adaptive-optics systems are computed by use of wavefront sensors, such as ShackHartmann or curvature sensors. In either case, the standard error of the phase estimates is proportional to the standard error of the measurements; but the error-propagation factors are different. We calculate the noise propagation for curvature and Shack-Hartmann sensors and their dependence on the number of sensors, $n$, on a circular aperture.

The key results are as follows:

1. If the sensor spacing is kept constant and the pupil is enlarged, the ratio increases as $\mathrm{n}^{0.4}$.

2. When more sensing elements are accommodated on the same aperture, it increases even faster, namely, proportional to $\mathrm{n}^{0.8}$. With large numbers of sensing elements, this increase can limit the applicability of curvature sensors.

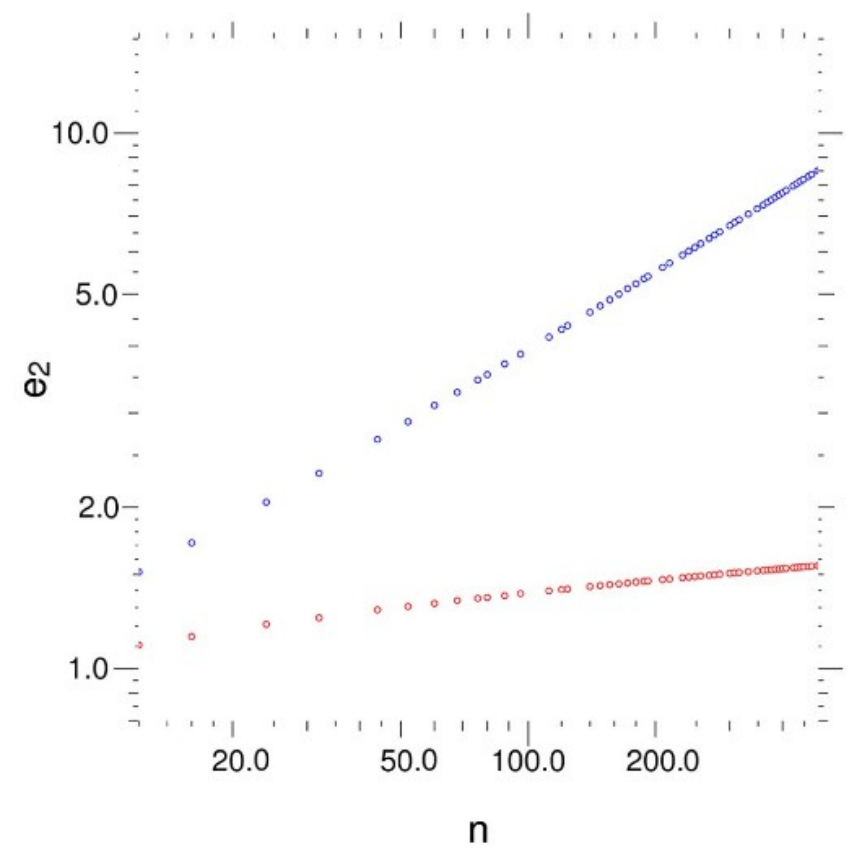

Figure 3: Comparison of the noise propagator for a curvature sensor (upper blue points) and a ShackHartmann sensor (lower red points). The $S$-H sensor's noise propagator increases as $\ln (n)$ while the curvature sensor's increases as $n$.

We note that the noise propagation error is just one of many errors in the overall system. In addition the fitting error (the residual wavefront after a perfect correction due to the limited number of correcting elements) is different for the two systems. Typically in a curvature adaptive optics system the correcting element is a curvature mirror and it has a smaller fitting error for a given number of correcting elements. However, in the low-light level regime the dominant error is the noise in the wavefront measurement (e.g. the noise propagation). 


\section{Conclusions:}

The conclusions of this study has important implications for the use of curvature sensors in a low-light level regime for space situational awareness.

1. In this regime (low light and quickly varying turbulence conditions) it is advantageous to adjust the curvature sensor's optical gain in real time. Significant gains in the performance of the system (Strehl ratio) are made in the low-light level regime.

2. We confirm that the noise propagator for curvature sensors grows linearly with the number of sensors in the case where the physical size of the sensor remains fixed (telescope aperture grows) and the optical gain/defocus distance is fixed. However, in the case of a fixed telescope aperture and decreasing subaperture physical size, there is no penalty in terms of the noise propagator for having more sensors. This relies on the fact that the curvature sensor can be implemented with a read-noise free detector.

3. Compared to a Shack-Hartmann sensor the curvature sensor noise propagator grows more rapidly with increasing number of sensors. In the low-light level regime, where measurement noise is the dominant error terms, this is problematic for curvature sensors. In addition, with the advent of CCD devices with no read noise (e.g. EMCCDs) the advantages of curvature sensors is diminished further.

\section{References:}

Kellerer, A., M Chun, and C Ftaclas (2010), “Automatic Sensitivity Adjustment for a Curvature Sensor", Applied Optics, 49 (31).

Kellerer, A. (2010), “Curvature Sensors: Noise and Its Propagation”, Journal of the Optical Society of America A, 27 (11).

Kellerer, Aglaé N, and Albrecht M Kellerer. (2011), “Error Propagation: a Comparison of ShackHartmann and Curvature Sensors", Journal of the Optical Society of America A 28 (5), 801. 